\title{
SEGETAL FLORA OF THE MIDDLE VISTULA RIVER VALLEY
}

\author{
Maria Ługowska \\ Department of Agricultural Ecology, University of Natural Sciences and Humanities \\ Prusa 14, 08-110 Siedlce, Poland \\ e-mail: lugowska@uph.edu.pl
}

Received: 05.05.2014

\begin{abstract}
The objective of the study was to describe the segetal flora of the Middle Vistula River Valley. A total of 367 species were recorded in spring and winter cereals, tuber crops, and stubble fields. Such floristic abundance may be due to the fact that the study area is located in the proximity of a river where semi-natural communities interact directly with communities inhabiting cultivated fields and that fields are fragmented and characterised by different habitats. There were more apophytes $(62 \%)$ than anthropophytes (38\%) in the flora studied. Meadow apophytes were the dominant native species $(35 \%)$ and archeophytes were the dominant anthropophytes $(69 \%)$. The analysis of the life spectrum revealed that there were more therophytes $(50 \%)$ than hemicryptophytes $(39 \%)$. What is more, non-perennial species constituted $56 \%$ and perennials $44 \%$ of the segetal flora established in the Middle Vistula River Valley. The large proportion of archeophytes (26\%) may indicate that traditional farming predominated in the study area.
\end{abstract}

Key words: segetal plants, anthropophytes, apophytes, agrocenoses, Middle Vistula River Valley.

\section{INTRODUCTION}

Soil and climatic conditions as well as anthropogenic factors affect the flora that establishes in cultivated fields. Changes in the floristic composition and structure of the communities have been observed for many years. Anthropogenic factors have the greatest influence on changes in segetal flora. They include the following: high mineral fertilisation, organic manuring, withdrawal of some plants from cultivation, the application of herbicides, reduced crop rotations, and the introduction of more efficient methods of seed material cleaning. Due to these factors, on the one hand, the area inhabited by specialised components of segetal flora is shrinking and some of them are even disappearing [1-3]; on the other hand, some species are increasing in number, their ranges are expanding, and they are penetrating new areas and habitats $[4,5]$.

Studies on segetal flora have been conducted in Poland for a very long time. However, there are some regions where no such research has been attempted so far. The Middle Vistula River Valley is an example of such a region and W ó j c i k [6] is the only author who has reported some data for this area.

\section{DESCRIPTION OF THE STUDY AREA}

The Mesoregion of the Middle Vistula River Valley is located in east-central Poland. In physicogeographical terms, it has been classified by $\mathrm{K}$ on d r a c ki [7] as a representative of the province of the Middle European Plain, sub-province of the Central Poland Lowlands, and macro-region of the Central Mazovian Lowland.

The Middle Vistula River Valley stretches from the Vistula gorge, runs through the Polish Uplands upstream of Puławy, and terminates at the valley's narrowing in Warsaw. The width of the valley is $10-12 \mathrm{~km}$ and its overall area is approximately $1350 \mathrm{~km}^{2}$. This section of the Vistula is around $120 \mathrm{~km}$ long; the river flows close to the left side of the valley and is quite wide (up to $1 \mathrm{~km}$ ). According to Woś [8], the Middle Vistula River Valley belongs to two climatic regions: the Central Mazovian Region and the Eastern Lesser Poland Region.

The Middle Vistula River Valley has slightly worse climatic conditions than the adjacent areas. It is located low relative to the riverbed, which, coupled with shallow groundwater table, contributes to higher variations in daily temperatures. Also, air humidity is higher, winds are stronger, and fogs occur on 60 days per year.

The study area is located on two terraces. Fragments of oxbows with many temporarily or permanently flooded hollows are located on the first terrace, whereas 
the second terrace is composed mainly of sands with no water flows.

The persistence of groundwater depends on relative heights, geology and water level in the river. In the proximity of the valley, water is present at a depth of 3 to $5 \mathrm{~m}$, whereas in the alluvial terrace groundwater occurs at a depth of 5 to $10 \mathrm{~m}$.

\section{MATERIALS AND METHODS}

Fieldwork was carried out from 2003 to 2008 at 117 localities of the Middle Vistula River Valley (Fig. 1). Floristic and phytosociological observations were conducted in cereals, tuber crops, and stubble fields, using the widely approved Braun-Blanquet method; in addition, numerous floristic lists were compiled.

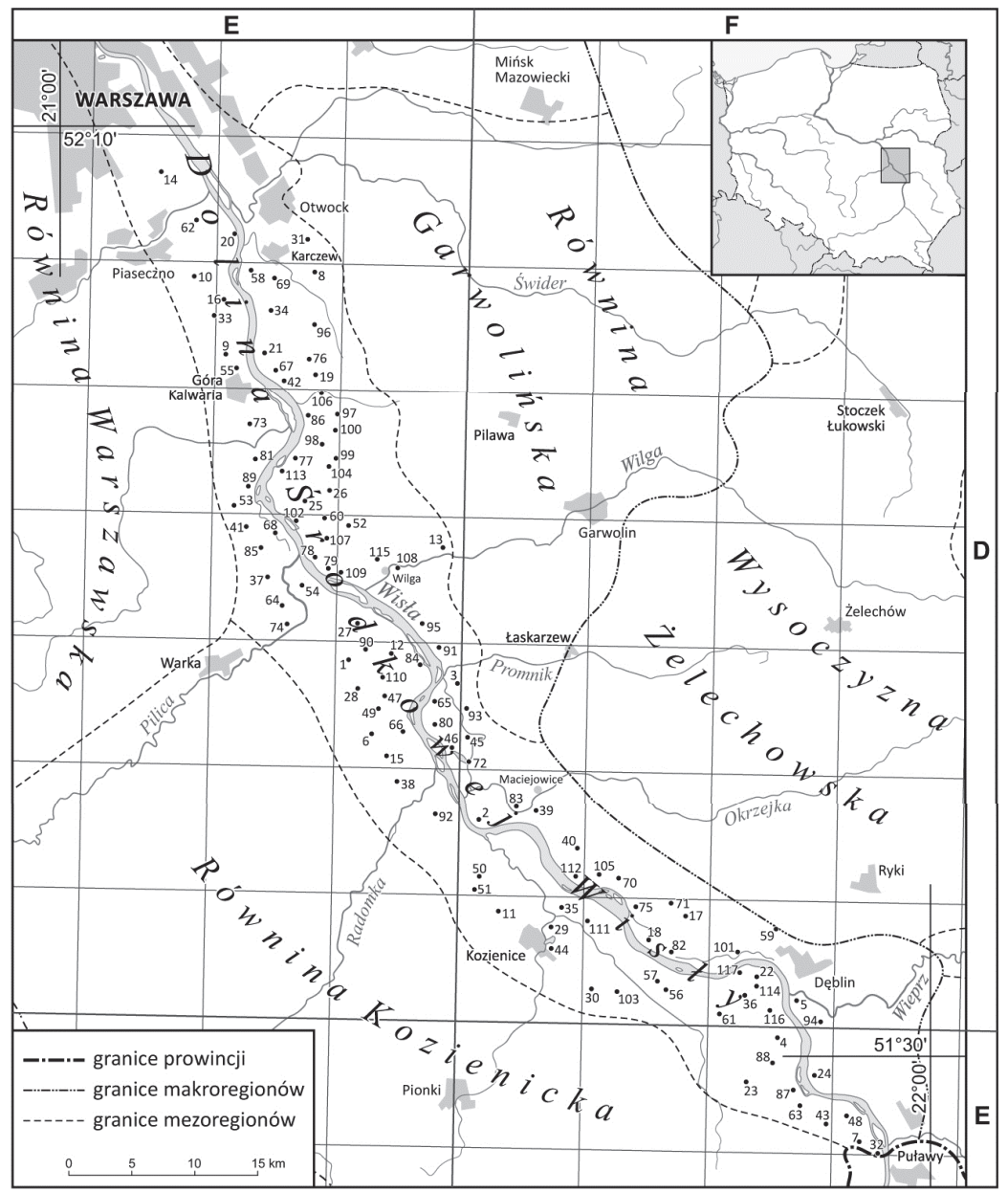

Fig 1. List of localities: 1 - Anielin, 2 - Antoniówka Świerżowska, 3 - Bączki, 4 - Borek, 5 - Borowa, 6 - Bożówka, 7 - Bronowice, 8 - Brzezinka, 9 - Brzeźce, 10 - Cieciszew, 11 - Chinów, 12 - Chmielew, 13 - Cyganówka, 14 - Czernidła, 15 - Dębowola, 16 - Dębówka, 17 - Długowola, 18 - Drahalica, 19 - Dziecinów, 20 - Gassy, 21 - Glinki, 22 - Głusiec, 23 - Gniewoszów, 24 - Gołąb, 25 - Goźlin, 26 - Goźlin Górny, 27 - Gruszczyce, 28 - Grzybów, 29 - Holendry Kozienickie, 30 - Janików, 31 - Janów, 32 - Jaroszyn, 33 - Kawęczyn, 34 - Kępa Nadbrzeska, 35 - Kępa Wólczyńska, 36 - Kępice. 37 - Klonowa Wola, 38 - Kłoda, 39 - Kochów, 40 - Kobylnica, 41 - Konary, 42 - Kosumce, 43 - Kowala, 44 - Kozienice, 45 - Kraski Dolne, 46 - Kraski Nowe, 47 - Kurki, 48 - Łęka, 49 - Magnuszew, 50 - Majdan, 51 - Majdany Kozienickie, 52 - Mariańskie Porzecze, 53 - Marynin, 54 - Mniszew, 55 - Moczydłów, 56 - Mozolice Duże, 57 - Mozolice Małe, 58 - Nadbrzeż, 59 - Nadwiślanka, 60 - Nieciecz, 61 - Opactwo, 62 - Opacz, 63 - Opatkowice, 64 - Ostrołęka, 65 - Ostrów (gm. Maciejowice), 66 - Ostrów (gm. Magnuszew), 67 - Ostrówek (gm. Karczew), 68 - Ostrówek (Warka), 69 - Otwock Wielki, 70 - Paprotnia, 71 - Pawłowice, 72 - Pasternik, 73 - Pęcław, 74 - Pilica, 75 - Piotrowice (gm. Stężyca), 76 - Piotrowice (gm. Karczew), 77 - Piwonin, 78 - Podole Nowe, 79 - Podole Stare, 80 - Podwierzbie, 81 - Potycz, 82 - Prażmów, 83 - Przewóz, 84 - Przewóz Stary, 85 - Przylot, 86 - Radwanków Szlachecki, 87 - Regów Nowy, 88 - Regów Stary, 89 - Rososzka, 90 - Rozniszew, 91 - Ruda Tarnowska, 92 - Ryczywół, 93 - Samogoszcz, 94 - Skoki, 95 - Skurcza, 96 - Sobiekursk, 97 - Sobienie Biskupie, 98 - Sobienie Jeziory, 99 - Sobienie Kiełczykowskie, 100 - Sobienie Szlacheckie, 101 - Stężyca, 102 - Szymanowice, 103 - Śmietanki, 104 - Śniadów, 105 - Turzyn Dworski, 106 - Warszawice, 107 - Wicie, 108 - Wilga, 109 - Wólka Gruszczyńska, 110 - Wólka Tarnowska, 111 - Wólka Turzyńska, 112 - Wróble Wargocin, 113 - Wysoczyn, 114 - Występ, 115 - Zakrzew, 116 - Zajezierze, 117 - Zbyczyn. 
The systematic layout follows Rutk ow ski [9] and species names follow Mirek et al. [10]. A multifaceted analysis of the flora was performed taking into account the geographical and historical affiliation of species, their persistence, biological type and frequency of occurrence. It was based on the works by the following authors: Rutk owski [9], Zają c [11], Jackowiak [12], Korniak [13], and R othmaler [14].

The incidence of species is based on the combined scale by J a sie wicz [15]: site = locality (1-3 sites - very rare, 4-9 sites - rare, 10-23 sites - rather rare, 24-46 sites - frequent, 47-69 - quite common, 70-93 - common, 94-117 sites - very common) (Table 1). The floristic lists also included crop self-seeders or crop plants running wild, both treated as ergasiophygophytes.

\section{RESULTS}

The flora that accompanies cereals and tuber crops and grows in stubble fields in the Middle Vistula River Valley encompasses 367 species of vascular plants representing 45 botanic families and 201 genera (Table 1). The following families had the most species: Asteraceae, Poaceae, Fabaceae, Brassicaceae, Caryophyllaceae and Scrophulariaceae. Species of the most abundant families were mostly apophytes and anthropophytes, apart from Brassicaceae and Chenopodiaceae where anthropophytes dominated (Table 1).

When the frequency of occurrence of individual species in the study area was considered, very rare and rare species were the most frequent group (Table 1). There were 223 taxons in this group, which accounts for $60.3 \%$ of segetal species in the study area. The group was dominated by species representing natural and semi-natural communities, including: Geranium pratense, Equisetum fluviatyle, Calystegia sepium, Lychnis flos-cuculi, Lysimachia nummularia, Torylis japonica, and Eryngium planum. Some species classified as very rare or rare are also rarely found in crop fields all over Poland, for example Neslia paniculata, Camelina sativa, Camelina microcarpa subsp. sylvestris, Valerianella dentata, Valerianella rimosa, Sherardia arvensis, and Fumaria vaillanti.

Table 1

Systematic list of species

\begin{tabular}{|c|c|c|c|c|c|}
\hline S.n. & Species & Origin & Persistence & Life form & Frequency \\
\hline \multicolumn{6}{|c|}{ Equisetaceae } \\
\hline 1. & Equisetum sylvaticum $\mathrm{L}$. & Ał & $\mathrm{W}$ & G & very rare \\
\hline 2. & E. arvense $\mathrm{L}$. & Ał & $\mathrm{W}$ & G & very common \\
\hline 3. & E. fluviatile $\mathrm{L}$. & Anw & $\mathrm{W}$ & $\mathrm{H}$ & very rare \\
\hline 4. & E. palustre L. & Ał & $\mathrm{W}$ & $\mathrm{G}$ & rare \\
\hline \multicolumn{6}{|c|}{ Cannabaceae } \\
\hline 5. & Humulus lupulus & $\mathrm{Al}$ & $\mathrm{W}$ & $\mathrm{H}$ & very rare \\
\hline \multicolumn{6}{|c|}{ Urticaceae } \\
\hline 6. & Urtica urens $\mathrm{L}$. & $\mathrm{Ar}$ & $\mathrm{K}$ & $\mathrm{T}$ & rare \\
\hline 7. & U. dioica $\mathrm{L}$. & $\mathrm{Al}$ & $\mathrm{W}$ & $\mathrm{G}(\mathrm{H})$ & rare \\
\hline \multicolumn{6}{|c|}{ Aristolochiaceae } \\
\hline 8. & Aristolochia clematitis L. & $\mathrm{Ar}$ & $\mathrm{W}$ & $\mathrm{H}$ & very rare \\
\hline \multicolumn{6}{|c|}{$\begin{array}{r}\text { Polygonaceae } \\
\end{array}$} \\
\hline 9. & Polygonum aviculare $\mathrm{L}$. & Anw & $\mathrm{K}$ & $\mathrm{T}$ & very common \\
\hline 10. & P. heterophyllum Lindm. Em.H Scholz & Anw & $\mathrm{K}$ & $\mathrm{T}$ & very rare \\
\hline 11. & P. bistorta $\mathrm{L}$ & Ał & $\mathrm{W}$ & G & rare \\
\hline 12. & P. amphibium $\mathrm{L}$. & Anw & $\mathrm{W}$ & G & rare \\
\hline 13. & P. hydropiper L. & Anw & K & $\mathrm{T}$ & frequent \\
\hline 14. & P. mite Schrank & Anw & $\mathrm{K}$ & $\mathrm{T}$ & very rare \\
\hline 15. & P. minus Huds & Anw & $\mathrm{K}$ & $\mathrm{T}$ & very rare \\
\hline 16. & $P$. persicaria $\mathrm{L}$. & Anw & $\mathrm{K}$ & $\mathrm{T}$ & common \\
\hline 17. & P. lapathifolium L. subsp. pallidum /With/Fr. & Anw & $\mathrm{K}$ & $\mathrm{T}$ & common \\
\hline 18. & P. lapathifolium L. subsp. lapathifolium & Anw & $\mathrm{K}$ & $\mathrm{T}$ & common \\
\hline 19. & Fallopia convolvulus /L./ Á. Löve & Ar & $\mathrm{K}$ & $\mathrm{T}$ & very common \\
\hline 20. & F. dumetorum (L.) Holub & $\mathrm{Al}(\mathrm{Az})$ & $\mathrm{K}$ & $\mathrm{T}$ & very rare \\
\hline 21. & Fagopyrum esculentum Gillib & Er & $\mathrm{K}$ & $\mathrm{T}$ & very rare \\
\hline
\end{tabular}




\begin{tabular}{|c|c|c|c|c|c|}
\hline 22. & Rumex acetosella $\mathrm{L}$. & Aps & $\mathrm{W}$ & G & quite common \\
\hline 23. & R. acetosa $\mathrm{L}$. & Ał & $\mathrm{W}$ & $\mathrm{H}$ & very rare \\
\hline 24. & R. obtusifolius L. & $\mathrm{Al}$ & W & G & very rare \\
\hline 25. & R. confertus Willd. & Ał & $\mathrm{W}$ & $\mathrm{H}$ & rare \\
\hline 26. & R. conglomeratus Murray & $\mathrm{Al}$ & W & G & very rare \\
\hline 27. & R. crispus $\mathrm{L}$. & At & W & G & rather rare \\
\hline \multicolumn{6}{|c|}{ Chenopodiaceae } \\
\hline 28. & Beta vulgaris $\mathrm{L}$. & $\mathrm{Er}$ & K & $\mathrm{T}$ & very rare \\
\hline 29. & Chenopodium glaucum $\mathrm{L}$. & Anw & K & $\mathrm{T}$ & very rare \\
\hline 30. & Ch. rubrum L. & Anw & K & $\mathrm{T}$ & very rare \\
\hline 31. & Ch. hybridum L. & $\mathrm{Ar}$ & K & $\mathrm{T}$ & very rare \\
\hline 32. & Ch. polyspermum $\mathrm{L}$. & Anw & K & $\mathrm{T}$ & rather rare \\
\hline 33. & Ch. suecicum Murr & $\mathrm{Ar}$ & K & $\mathrm{T}$ & very rare \\
\hline 34. & Ch. strictum Roth & Ep & $\mathrm{K}$ & $\mathrm{T}$ & very rare \\
\hline 35. & Ch. album $\mathrm{L}$. & Anw & K & $\mathrm{T}$ & very common \\
\hline 36. & Atriplex nitens Schkuhr & $\mathrm{Ar}$ & K & $\mathrm{T}$ & very rare \\
\hline 37. & A. tatarica $\mathrm{L}$. & Ef & K & $\mathrm{T}$ & very rare \\
\hline 38. & A. patula $\mathrm{L}$. & $\mathrm{Ar}$ & $\mathrm{K}$ & $\mathrm{T}$ & rather rare \\
\hline \multicolumn{6}{|c|}{ Amaranthaceae } \\
\hline 39. & Amaranthus retroflexus $\mathrm{L}$. & Ep & K & $\mathrm{T}$ & quite common \\
\hline 40. & A. lividus & Ep & $\mathrm{K}$ & $\mathrm{T}$ & rather rare \\
\hline \multicolumn{6}{|c|}{ Caryophyllaceae } \\
\hline 41. & Arenaria serpyllifolia $\mathrm{L}$. & Aps & K & $\mathrm{T}$ & frequent \\
\hline 42. & Stellaria nemorum L. & $\mathrm{Al}$ & W & $\mathrm{H}$ & very rare \\
\hline 43. & S. media /L./ Vill. & Ał & K & $\mathrm{T}$ & very common \\
\hline 44. & S. graminea $\mathrm{L}$. & Ał & W & $\mathrm{H}$ & rather rare \\
\hline 45. & Cerastium arvense L. s.s. & Aps & K & $\mathrm{H}$ & rare \\
\hline 46. & C. holosteoides Fr. em. Hyl. & Ał & K & $\mathrm{H}$ & frequent \\
\hline 47. & C. semidecandrum $\mathrm{L}$. & Amk & K & $\mathrm{T}$ & very rare \\
\hline 48. & Sagina procumbens $\mathrm{L}$. & Ał & $\mathrm{W}$ & $\mathrm{H}$ & rather rare \\
\hline 49 & Scleranthus annuиs L. & $\mathrm{Ar}$ & K & $\mathrm{T}$ & quite common \\
\hline 50. & S. perennis L. & Aps & W & $\mathrm{H}$ & rare \\
\hline 51. & Herniaria hirsuta $\mathrm{L}$. & Aps & K & $\mathrm{T}$ & rare \\
\hline 52. & Spergula arvensis $\mathrm{L}$. & $\mathrm{Ar}$ & K & $\mathrm{T}$ & quite common \\
\hline 53. & S. morisonii Boreau & Aps & K & $\mathrm{T}$ & rather rare \\
\hline 54. & Spergularia rubra /L./J. Presl et C. Presl & Anw & K & $\mathrm{T}$ & frequent \\
\hline 55. & Lychnis flos-cuculi L. & Ał & W & $\mathrm{H}$ & very rare \\
\hline 56. & Agrostemma githago $\mathrm{L}$. & $\mathrm{Ar}$ & K & $\mathrm{T}$ & frequent \\
\hline 57. & Melandrium album /Mill./ Garcke & Ał & K & $\mathrm{T}$ & quite common \\
\hline 58. & M. noctiflorum /L./ Fr. & $\mathrm{Ar}$ & K & $\mathrm{T}$ & frequent \\
\hline 59. & Gypsophila muralis L. & Anw & K & $\mathrm{T}$ & frequent \\
\hline 60. & Saponaria officinalis L. & Anw & $\mathrm{W}$ & G & rare \\
\hline 61. & Silene vulgaris /Moench/ Garcke & Amk & $\mathrm{W}$ & $\mathrm{H}$ & rare \\
\hline \multicolumn{6}{|c|}{ Ranunculaceae } \\
\hline 62. & Consolida regalis Gray & $\mathrm{Ar}$ & K & $\mathrm{T}$ & frequent \\
\hline 63. & Ranunculus flammula $\mathrm{L}$. & Anw & W & $\mathrm{H}$ & very rare \\
\hline 64. & R. bulbosus L. & Amk & W & $\mathrm{H}$ & rare \\
\hline 65. & R. arvensis $\mathrm{L}$. & $\mathrm{Ar}$ & $\mathrm{K}$ & $\mathrm{T}$ & very rare \\
\hline 66. & R. repens $\mathrm{L}$. & Ał & $\mathrm{W}$ & $\mathrm{H}$ & frequent \\
\hline 67. & R. sardous Crantz & Ał & $\mathrm{W}$ & $\mathrm{H}$ & rare \\
\hline 68. & R. acris L. S. STR. & Ał & $\mathrm{W}$ & $\mathrm{H}$ & very rare \\
\hline 69. & Myosurus minimus L. & Anw & $\mathrm{K}$ & $\mathrm{T}$ & rare \\
\hline
\end{tabular}


Papaveraceae

\begin{tabular}{|c|c|c|c|c|c|}
\hline 70. & Papaver sommniferum $\mathrm{L}$. & $\mathrm{Er}$ & $\mathrm{K}$ & $\mathrm{T}$ & very rare \\
\hline 71. & P. argemone $\mathrm{L}$. & $\mathrm{Ar}$ & $\mathrm{K}$ & $\mathrm{T}$ & rather rare \\
\hline 72. & P. dubium L. & $\mathrm{Ar}$ & K & $\mathrm{T}$ & rather rare \\
\hline 73. & P. rhoeas L. & $\mathrm{Ar}$ & $\mathrm{K}$ & $\mathrm{T}$ & common \\
\hline 74. & Chelidonium majus L. & $\mathrm{Al}$ & W & $\mathrm{H}$ & rare \\
\hline \multicolumn{6}{|c|}{ Fumariaceae } \\
\hline 75. & Corydalis solida $\mathrm{Sm}$. & $\mathrm{Al}$ & W & $\mathrm{G}$ & very rare \\
\hline 76. & Fumaria officinalis L. & $\mathrm{Ar}$ & $\mathrm{K}$ & $\mathrm{T}$ & rather rare \\
\hline 77. & F. valliantii Loisel & $\mathrm{Ar}$ & $\mathrm{K}$ & $\mathrm{T}$ & very rare \\
\hline \multicolumn{6}{|c|}{ Brassicaceae } \\
\hline 78. & Sisymbrium officinale /L./ Scop. & $\mathrm{Ar}$ & $\mathrm{K}$ & $\mathrm{T}$ & rather rare \\
\hline 79. & S. loeselii L. & Ep & $\mathrm{K}$ & $\mathrm{H}$ & rare \\
\hline 80. & Descurainia sophia /L./ Webb. ex Prantl. & $\mathrm{Ar}$ & $\mathrm{K}$ & $\mathrm{T}$ & rather rare \\
\hline 81. & Arabidopsis thaliana /L./ Heynh. & Aps & $\mathrm{K}$ & $\mathrm{T}$ & frequent \\
\hline 82. & Erysimum cheiranthoides L. & $\mathrm{Ar}$ & $\mathrm{K}$ & $\mathrm{T}$ & frequent \\
\hline 83. & Rorippa palustris /L./ Besser & Anw & $\mathrm{K}$ & $\mathrm{T}$ & frequent \\
\hline 84. & R. sylvestris /L./ Besser & Anw & W & $\mathrm{H}$ & quite common \\
\hline 85. & R. austriaca /Crantz/ Besser & $\mathrm{Kn}$ & W & $\mathrm{H}$ & rather rare \\
\hline 86. & R. amphibia /L./ Besser & Anw & W & G & rare \\
\hline 87. & Armoracia rusticana P.Gaertn., B. Mey. et Scherb. & $\mathrm{Ar}$ & $\mathrm{W}$ & $\mathrm{G}$ & rare \\
\hline 88. & Cardaminopsis arenosa (L.) Hayek & Aps & $\mathrm{K}$ & $\mathrm{T}$ & rather rare \\
\hline 89. & Alyssum alyssoides (L.) L. & Amk & $\mathrm{K}$ & $\mathrm{T}$ & very rare \\
\hline 90. & Berteroa incana /L./ DC & Amk & $\mathrm{K}$ & $\mathrm{T}$ & rare \\
\hline 91. & Erophila verna /L./ Chevall. & Aps & $\mathrm{K}$ & $\mathrm{T}$ & rather rare \\
\hline 92. & $\begin{array}{l}\text { Camelina microcarpa subsp. sylvestris (Wallr.) } \\
\text { Hiitonen }\end{array}$ & Amk & $\mathrm{K}$ & $\mathrm{T}$ & rare \\
\hline 93. & C. sativa (L.) Crantz & Ar & $\mathrm{K}$ & $\mathrm{T}$ & very rare \\
\hline 94. & Neslia paniculata (L.) Desv. & $\mathrm{Ar}$ & $\mathrm{K}$ & $\mathrm{T}$ & very rare \\
\hline 95. & Capsella bursa-pastoris/L./ Medik. & $\mathrm{Ar}$ & $\mathrm{K}$ & $\mathrm{T}$ & very common \\
\hline 96. & Teesdalea nudicaulis /L./ R. Br. & Aps & $\mathrm{K}$ & $\mathrm{T}$ & rather rare \\
\hline 97. & Thlaspi arvense $\mathrm{L}$. & $\mathrm{Ar}$ & $\mathrm{K}$ & $\mathrm{T}$ & frequent \\
\hline 98. & Lepidium ruderale $\mathrm{L}$. & $\mathrm{Ar}$ & $\mathrm{K}$ & $\mathrm{T}$ & rare \\
\hline 99. & Brassica napus L. subsp. napus & $\mathrm{Er}$ & $\mathrm{K}$ & $\mathrm{T}$ & very rare \\
\hline 100. & B. rapa L. subsp. oleifera & Er & K & $\mathrm{T}$ & very rare \\
\hline 101. & Sinapis arvensis $\mathrm{L}$. & $\mathrm{Ar}$ & $\mathrm{K}$ & $\mathrm{T}$ & rather rare \\
\hline 102. & S. alba L. & $\mathrm{Er}$ & $\mathrm{K}$ & $\mathrm{T}$ & rare \\
\hline 103. & Raphanus raphanistrum $\mathrm{L}$. & $\mathrm{Ar}$ & $\mathrm{K}$ & $\mathrm{T}$ & quite common \\
\hline \multicolumn{6}{|c|}{ Crassulaceae } \\
\hline 104. & Sedum maximum /L./ Hoffm. & Amk & W & $\mathrm{G}$ & very rare \\
\hline 105. & S. acre L. & Amk & $\mathrm{W}$ & $\mathrm{H}$ & very rare \\
\hline \multicolumn{6}{|c|}{ Rosaceae } \\
\hline 106. & Filipendula ulmaria (L.) Maxim. & Ał & $\mathrm{W}$ & $\mathrm{H}$ & very rare \\
\hline 107. & Rubus caesius L. & $\mathrm{Al}$ & W & $\mathrm{Ch}$ & rare \\
\hline 108. & Sanguisorba officinalis L. & Ał & W & $\mathrm{H}$ & very rare \\
\hline 109. & Geum urbanum L. & $\mathrm{Al}$ & W & $\mathrm{H}$ & rare \\
\hline 110. & Potentilla anserina $\mathrm{L}$. & Ał & W & $\mathrm{H}$ & frequent \\
\hline 111. & P. reptans $\mathrm{L}$. & Ał & W & $\mathrm{H}$ & rare \\
\hline 112. & P. norvegica $\mathrm{L}$. & Anw & $\mathrm{K}$ & $\mathrm{T}$ & rare \\
\hline 113. & P. arenaria Borkh. & Amk & W & $\mathrm{H}$ & very rare \\
\hline 114. & P. collina Wibel s.s. & Anw & $\mathrm{W}$ & $\mathrm{H}$ & very rare \\
\hline
\end{tabular}




\begin{tabular}{|c|c|c|c|c|c|}
\hline 115. & P. argentea L.s.s & Amk & $\mathrm{W}$ & $\mathrm{H}$ & rare \\
\hline 116. & Alchemilla monticola Opiz & At & W & $\mathrm{H}$ & very rare \\
\hline 117. & Aphanes arvensis $\mathrm{L}$. & $\mathrm{Ar}$ & $\mathrm{K}$ & $\mathrm{T}$ & very rare \\
\hline \multicolumn{6}{|c|}{ Fabaceae } \\
\hline 118. & Lupinus luteus $\mathrm{L}$. & Er & $\mathrm{K}$ & $\mathrm{T}$ & rare \\
\hline 119. & Galega officinalis $\mathrm{L}$. & Ef & W & $\mathrm{H}$ & very rare \\
\hline 120. & Astragalus glycyphyllos L. & $\mathrm{Al}$ & W & $\mathrm{H}$ & very rare \\
\hline 121. & Vicia sepium $\mathrm{L}$. & $\mathrm{Al}$ & W & $\mathrm{H}$ & very rare \\
\hline 122. & V. sativa $\mathrm{L}$. & $\mathrm{Ar}$ & $\mathrm{K}$ & $\mathrm{T}$ & rather rare \\
\hline 123. & V. angustifolia $\mathrm{L}$. & $\mathrm{Ar}$ & K & $\mathrm{T}$ & common \\
\hline 124. & V. hirsuta /L./ S. F. Gray. & $\mathrm{Ar}$ & $\mathrm{K}$ & $\mathrm{T}$ & very common \\
\hline 125. & V. tetrasperma /L./ Schreb. & $\mathrm{Ar}$ & K & $\mathrm{T}$ & common \\
\hline 126. & V. villosa Roth & $\mathrm{Ar}$ & K & $\mathrm{T}$ & quite common \\
\hline 127. & V. cracca L. & At & W & $\mathrm{H}$ & frequent \\
\hline 128. & Lathyrus pratensis $\mathrm{L}$. & At & W & $\mathrm{H}$ & rare \\
\hline 129. & L. tuberosus L. & $\mathrm{Ar}$ & W & G & quite common \\
\hline 130. & Pisum sativum $\mathrm{L}$. & Er & $\mathrm{K}$ & $\mathrm{T}$ & rare \\
\hline 131. & P. sativum L. subsp. arvense (L.)Asch\&Graebn. & Er & K & $\mathrm{T}$ & rare \\
\hline 132. & Melilotus alba $\mathrm{L}$. & Amk & K & $\mathrm{T}$ & very rare \\
\hline 133. & M. officinalis (L.) Pall. & $\mathrm{Al}$ & $\mathrm{K}$ & $\mathrm{T}$ & rare \\
\hline 134. & Medicago sativa L. S. STR. & $\mathrm{Er}$ & $\mathrm{W}$ & $\mathrm{H}$ & very rare \\
\hline 135. & M. falcata $\mathrm{L}$. & Amk & $\mathrm{W}$ & $\mathrm{H}$ & rare \\
\hline 136. & M. lupulina $\mathrm{L}$. & Amk & K & $\mathrm{T}$ & frequent \\
\hline 137. & Trifolium dubium Sibth. & Ał & $\mathrm{K}$ & $\mathrm{T}$ & rare \\
\hline 138. & T. campestre Schreb. & Ał & K & $\mathrm{T}$ & rare \\
\hline 139. & T. repens $\mathrm{L}$. & Ał & W & $\mathrm{H}$ & common \\
\hline 140. & T. hybridum L. & Ał & $\mathrm{W}$ & $\mathrm{H}$ & rare \\
\hline 141. & T. rubens $L$. & Aps & $\mathrm{W}$ & $\mathrm{H}$ & very rare \\
\hline 142. & T. medium $\mathrm{L}$. & $\mathrm{Az}$ & W & $\mathrm{H}$ & very rare \\
\hline 143. & T. pratense $\mathrm{L}$. & $\mathrm{Al}$ & K & $\mathrm{T}$ & frequent \\
\hline 144. & T. arvense $\mathrm{L}$. & Aps & K & $\mathrm{T}$ & rather rare \\
\hline 145. & Lotus corniculatus L. & Al & K & $\mathrm{H}$ & rather rare \\
\hline 146. & Ornithopus sativus Brot. & Er & $\mathrm{K}$ & $\mathrm{T}$ & very rare \\
\hline \multicolumn{6}{|c|}{ Oxalidaceae } \\
\hline 147. & Oxalis fontana Bunge & Ep & $\mathrm{W}$ & $\mathrm{H}$ & common \\
\hline 148. & O. acetosella $\mathrm{L}$. & $\mathrm{Al}$ & $\mathrm{W}$ & G & rare \\
\hline \multicolumn{6}{|c|}{ Geraniaceae } \\
\hline 149. & Geranium pratense $\mathrm{L}$. & Ał & $\mathrm{W}$ & $\mathrm{H}$ & rare \\
\hline 150. & G. dissectum L. & $\mathrm{Ar}$ & K & $\mathrm{T}$ & very rare \\
\hline 151. & G. pusillum Burm. f. ex L. & $\mathrm{Ar}$ & K & $\mathrm{T}$ & frequent \\
\hline 152. & Erodium cicutarium /L./ L' Hér. & $\mathrm{Ar}$ & $\mathrm{K}$ & $\mathrm{T}$ & frequent \\
\hline \multicolumn{6}{|c|}{ Linaceae } \\
\hline 153. & Linum usitatissimum $\mathrm{L}$. & Er & $\mathrm{K}$ & $\mathrm{T}$ & very rare \\
\hline 154. & Radiola linoides Rothm. & Anw & $\mathrm{K}$ & $\mathrm{T}$ & rare \\
\hline \multicolumn{6}{|c|}{$\begin{array}{ll}\text { Euphorbiaceae } \\
\end{array}$} \\
\hline 155. & Euphorbia helioscopia L. & $\mathrm{Ar}$ & $\mathrm{K}$ & $\mathrm{T}$ & common \\
\hline 156. & E. palustris L. & Anw & W & $\mathrm{H}$ & very rare \\
\hline 157. & E. exigua $\mathrm{L}$. & $\mathrm{Ar}$ & K & $\mathrm{T}$ & rather rare \\
\hline 158. & E. peplus $\mathrm{L}$. & $\mathrm{Ar}$ & K & $\mathrm{T}$ & rare \\
\hline 159. & E. cyparissias $\mathrm{L}$. & Amk & $\mathrm{W}$ & G & rare \\
\hline
\end{tabular}




\begin{tabular}{|c|c|c|c|c|c|}
\hline 160. & E. lucida Waldst. \& Kit. & Anw & $\mathrm{W}$ & $\mathrm{H}$ & very rare \\
\hline 161. & E. esula L. & Amk & $\mathrm{K}$ & $\mathrm{H}$ & rather rare \\
\hline \multicolumn{6}{|c|}{ Balsaminaceae } \\
\hline 162. & Impatiens parviflora DC. & Er & $\mathrm{K}$ & $\mathrm{T}$ & very rare \\
\hline \multicolumn{6}{|c|}{ Malvaceae } \\
\hline 163. & Malva pusilla Sm. & $\mathrm{Ar}$ & $\mathrm{K}$ & $\mathrm{H}$ & very rare \\
\hline 164. & M. neglecta Wallr. & $\mathrm{Ar}$ & $\mathrm{K}$ & $\mathrm{H}$ & rather rare \\
\hline 165. & M. sylvestris L. & $\mathrm{Ar}$ & $\mathrm{K}$ & $\mathrm{H}$ & rare \\
\hline \multicolumn{6}{|c|}{ Clusiaceae } \\
\hline 166. & Hypericum humifusum L. & Aps & W & $\mathrm{T}(\mathrm{H})$ & rather rare \\
\hline 167. & H. perforatum L. & Ał & $\mathrm{W}$ & $\mathrm{H}$ & rather rare \\
\hline \multicolumn{6}{|c|}{ Violaceae } \\
\hline 168. & Viola arvensis Murray & $\mathrm{Ar}$ & $\mathrm{K}$ & $\mathrm{T}$ & very common \\
\hline 169. & V. tricolor L. S. STR. & Aps & $\mathrm{K}$ & $\mathrm{T}$ & rare \\
\hline \multicolumn{6}{|c|}{ Lythraceae } \\
\hline 170. & Lythrum salicaria $\mathrm{L}$. & Ał & W & $\mathrm{H}$ & very rare \\
\hline 171. & Peplis portula $\mathrm{L}$. & Anw & $\mathrm{K}$ & $\mathrm{T}$ & rare \\
\hline \multicolumn{6}{|c|}{ Onagraceae } \\
\hline 172. & Oenothera biennis L. S. STR. & Aps & $\mathrm{K}$ & $\mathrm{T}$ & rare \\
\hline 173. & Chamaenerion angustifolium (L.)Scop. & $\mathrm{Az}$ & W & $\mathrm{H}$ & very rare \\
\hline 174. & Epilobium parviflorum & Anw & W & $\mathrm{H}$ & rather rare \\
\hline 175. & E. roseum Schreb. & Anw & $\mathrm{W}$ & $\mathrm{H}$ & rare \\
\hline \multicolumn{6}{|c|}{ Apiaceae } \\
\hline 176. & Eryngium planum $\mathrm{L}$. & Aps & $\mathrm{W}$ & $\mathrm{H}$ & very rare \\
\hline 177. & Anthriscus sylvestris (L.) Hoffm. & $\mathrm{Al}$ & W & $\mathrm{H}$ & very rare \\
\hline 178. & Pimpinella saxifraga $\mathrm{L}$. & Amk & $\mathrm{W}$ & $\mathrm{H}$ & rather rare \\
\hline 179. & Aegopodium podagraria $\mathrm{L}$. & Amk & $\mathrm{W}$ & $\mathrm{H}$ & rare \\
\hline 180. & Aethusa cynapium $\mathrm{L}$. & $\mathrm{Ar}$ & $\mathrm{K}$ & $\mathrm{T}$ & frequent \\
\hline 181. & Anethum graveolens $\mathrm{L}$. & Er & $\mathrm{K}$ & $\mathrm{T}$ & very rare \\
\hline 182 & Falcaria vulgaris Bernh & Amk & W & $\mathrm{H}$ & rare \\
\hline 183. & Carum carvi $\mathrm{L}$. & $\mathrm{Al}$ & $\mathrm{K}$ & $\mathrm{T}$ & very rare \\
\hline 184. & Pastinaca sativa L. s.s. & Ał & $\mathrm{K}$ & $\mathrm{H}$ & rare \\
\hline 185. & Heracleum sphondylium L. S.STR. & Ał & W & $\mathrm{H}$ & very rare \\
\hline 186. & H. sibiricum $\mathrm{L}$. & Al & W & $\mathrm{H}$ & very rare \\
\hline 187. & Torilis japonica (Houtt.) DC. & $\mathrm{Az}$ & $\mathrm{K}$ & $\mathrm{T}$ & very rare \\
\hline 188. & Daucus carota L. & Ar & $\mathrm{K}$ & $\mathrm{T}$ & frequent \\
\hline \multicolumn{6}{|c|}{ Primulaceae } \\
\hline 189. & Lysimachia nummularia $\mathrm{L}$. & Al & $\mathrm{W}$ & G & rare \\
\hline 190. & L. vulgaris $\mathrm{L}$. & Ał & $\mathrm{W}$ & G & rare \\
\hline 191. & Centunculus minimus $\mathrm{L}$. & $\mathrm{Ar}$ & $\mathrm{K}$ & $\mathrm{T}$ & rare \\
\hline 192. & Anagallis arvensis L. & $\mathrm{Ar}$ & $\mathrm{K}$ & $\mathrm{T}$ & common \\
\hline \multicolumn{6}{|c|}{ Plumbaginaceae } \\
\hline 193. & Armeria maritima (Mill.)Willd. & Aps & $\mathrm{W}$ & $\mathrm{H}$ & very rare \\
\hline \multicolumn{6}{|c|}{ Gentianaceae } \\
\hline 194. & Centaurium pulchellum (Sw.) Druce & Al & $\mathrm{K}$ & $\mathrm{T}$ & rather rare \\
\hline \multicolumn{6}{|c|}{ Rubiaceae } \\
\hline 195. & Sherardia arvensis $\mathrm{L}$. & $\mathrm{Al}$ & $\mathrm{K}$ & $\mathrm{T}$ & rare \\
\hline 196. & Galium palustre $\mathrm{L}$. & Anw & W & $\mathrm{H}$ & very rare \\
\hline 197. & G. aparine $\mathrm{L}$. & $\mathrm{Al}$ & $\mathrm{K}$ & $\mathrm{T}$ & common \\
\hline 198. & G. spurium L. & $\mathrm{Ar}$ & $\mathrm{K}$ & $\mathrm{T}$ & rare \\
\hline
\end{tabular}




\begin{tabular}{llllll}
\hline 199. & G. odoratum (L.) Scop. & Al & W & H & very rare \\
200. & G. verum L. & Al & W & G & rare \\
201. & G. mollugo L. & Al & W & G & rare \\
\hline
\end{tabular}

\section{Convolvulaceae}

202. Cuscuta campestris Yuncker

203. Calystegia sepium L.

204. Convolvulus arvensis L.

\section{Hydrophyllaceae}

205. Phacelia tanacetifolia Benth

\section{Boraginaceae}

206. Lithospermum arvense $\mathrm{L}$.

207. Echium vulgare L.

208. Symphytum officinale L.

209. Anchusa officinalis $L$.

210. A. arvensis /L./ M. Bieb.

211. Myosotis arvensis /L./ Hill.

212. M. stricta Link ex Roem. i Schult

\section{Lamiaceae}

213. Ajuga reptans $L$.

214. Galeopsis ladanum L.

215. G. speciosa Mill.

216. G. pubescens Besser

217. G. tetrahit L.

218. G. bifida Boenn.

219. Lamium album $\mathrm{L}$.

220. L. amplexicaule L.

221. L. purpureum L.

222. Leonurus cardiaca L.

223. Ballota nigra L.

224. Stachys annua L.

225. S. palustris L.

226. Glechoma hederacea $\mathrm{L}$.

227. Prunella vulgaris L.

228. Thymus serpyllum L. Emend. Fr.

229. Lycopus europeus L.

230. Mentha arvensis L

231. Elsholtzia ciliata (Thunb.) Hyl.

\section{Solanaceae}

232. Solanum nigrum L. em. Mill.

233. Datura stramonium L.

\section{Scrophulariaceae}

234. Verbascum thapsus

235. V. nigrum L.

236. Scrophularia nodosa $\mathrm{L}$

237. Chaenorhinum minus (L.) Lange

238. Linaria vulgaris (L.) Mill.

239. Kickxia elatine (L.) Dumort.

240. Veronica spicata $\mathrm{L}$.

241. V. serpyllifolia $\mathrm{L}$.

242. V. triphyllos L.

$\begin{array}{cccl}\text { Ep } & \text { K } & \text { T } & \text { very rare } \\ \text { Az } & \text { W } & \text { H } & \text { very rare } \\ \text { Amk } & \text { W } & \text { G } & \text { very common }\end{array}$

very rare

rather rare

$\begin{array}{clll}\text { Ar } & \mathrm{K} & \mathrm{T} & \text { rather rare } \\ \text { Amk } & \mathrm{K} & \mathrm{H} & \text { rare } \\ \text { Anw(1) } & \mathrm{W} & \mathrm{G} & \text { frequent } \\ \text { Amk } & \mathrm{K} & \mathrm{H} & \text { rare } \\ \mathrm{Ar} & \mathrm{K} & \mathrm{T} & \text { frequent } \\ \mathrm{Ar} & \mathrm{K} & \mathrm{T} & \text { very common } \\ \text { Aps } & \mathrm{K} & \mathrm{T} & \text { rather rare }\end{array}$

$\mathrm{Az}$

Ar

Al

$\mathrm{Al}$

Al

$\mathrm{Al}$

Ar

$\mathrm{Ar}$

$\mathrm{Ar}$

$\mathrm{Ar}$

Ar

Ar

Anw

At

Ał

Aps

Anw

Anw

Ep
K

W

W
K

K

K

K

K

K

K

K

K

\section{K}

W

W

K

W

W

W

W

W

W

K

Ar
Ep

$\mathrm{K}$
$\mathrm{K}$

K

T

frequent

very rare

very rare

very rare

rather rare

frequent

rare

very rare

quite common

quite common

very rare

rare

rare

quite common frequent

rare

very rare

very rare quite common very rare

\begin{tabular}{ccll} 
Amk & K & T & very rare \\
$\mathrm{Al}$ & $\mathrm{K}$ & $\mathrm{H}$ & very rare \\
$\mathrm{Al}$ & $\mathrm{W}$ & $\mathrm{H}$ & rare \\
Amk & $\mathrm{K}$ & $\mathrm{T}$ & frequent \\
Aps & $\mathrm{W}$ & $\mathrm{G}$ & rather rare \\
$\mathrm{Ar}$ & $\mathrm{K}$ & $\mathrm{T}$ & rather rare \\
Amk & $\mathrm{W}$ & $\mathrm{H}$ & very rare \\
$\mathrm{Al}$ & $\mathrm{W}$ & $\mathrm{H}$ & rare \\
$\mathrm{Ar}$ & $\mathrm{K}$ & $\mathrm{T}$ & rather rare \\
\hline
\end{tabular}




\begin{tabular}{|c|c|c|c|c|c|}
\hline 243. & V. arvensis $\mathrm{L}$. & $\mathrm{Ar}$ & $\mathrm{K}$ & $\mathrm{T}$ & common \\
\hline 344. & V. verna $\mathrm{L}$. & Amk & $\mathrm{K}$ & $\mathrm{T}$ & rather rare \\
\hline 245. & V. dillenii Cranz. & Aps & $\mathrm{K}$ & $\mathrm{T}$ & rather rare \\
\hline 246. & V. hederifolia L. s.s. & $\mathrm{Ar}$ & $\mathrm{K}$ & $\mathrm{T}$ & rare \\
\hline 247. & $V$. persica Poir. & Ep & $\mathrm{K}$ & $\mathrm{T}$ & common \\
\hline 248. & V. agrestis L. & $\mathrm{Ar}$ & $\mathrm{K}$ & $\mathrm{T}$ & frequent \\
\hline 249. & $V$. polita Fr. & $\mathrm{Ar}$ & $\mathrm{K}$ & $\mathrm{T}$ & frequent \\
\hline 250. & V. opaca Fr. & $\mathrm{Ar}$ & $\mathrm{K}$ & $\mathrm{T}$ & rather rare \\
\hline 251. & V. anagallis-aquatica $\mathrm{L}$. & Anw & W & Hy & very rare \\
\hline 252. & V. chamaedrys L. S. STR. & Ał & W & $\mathrm{H}$ & rare \\
\hline 253. & Odontites serotina (Lam.) Rchb. & Ał & $\mathrm{K}$ & $\mathrm{T}$ & very rare \\
\hline 254. & Rhinanthus serotinus /Schönh./ Oborný & Ar & $\mathrm{K}$ & $\mathrm{T}$ & rare \\
\hline \multicolumn{6}{|c|}{ Plantaginaceae } \\
\hline 255. & Plantago media L. & Ał & $\mathrm{W}$ & $\mathrm{H}$ & rare \\
\hline 256. & P. major L. & $\mathrm{Al}$ & W & $\mathrm{H}$ & common \\
\hline 257. & P. intermedia Gilib. & Anw & W & $\mathrm{H}$ & quite common \\
\hline 258. & P. lanceolata $\mathrm{L}$. & Ał & $\mathrm{W}$ & $\mathrm{H}$ & frequent \\
\hline \multicolumn{6}{|c|}{ Valerianaceae } \\
\hline 259. & Valerianella rimosa Bastard & $\mathrm{Ar}$ & $\mathrm{K}$ & $\mathrm{T}$ & very rare \\
\hline 260. & V. dentata (L.) Pollich & $\mathrm{Ar}$ & $\mathrm{K}$ & $\mathrm{T}$ & rare \\
\hline \multicolumn{6}{|c|}{ Dipsacaceae } \\
\hline 261. & Knautia arvensis /L./ J.M. Coult. & $\mathrm{Al}$ & $\mathrm{W}$ & $\mathrm{H}$ & rare \\
\hline \multicolumn{6}{|c|}{ Campanulaceae } \\
\hline 262. & Campanula patula Griseb. & Ał & W & $\mathrm{H}$ & very rare \\
\hline 263. & C. rapunculoides $\mathrm{L}$. & Amk & W & $\mathrm{G}$ & rare \\
\hline 264. & Jasione montana $\mathrm{L}$. & Aps & $\mathrm{K}$ & $\mathrm{H}$ & very rare \\
\hline \multicolumn{6}{|c|}{ Asteraceae } \\
\hline 265. & Solidago virgaurea $\mathrm{L}$. & Al & W & $\mathrm{H}$ & rare \\
\hline 266. & S. gigantea Aiton & Ep & $\mathrm{W}$ & $\mathrm{H}$ & rare \\
\hline 267. & S. canadensis L. & Ep & $\mathrm{W}$ & $\mathrm{H}$ & rare \\
\hline 268. & Bellis perennis L. & Al & W & $\mathrm{H}$ & very rare \\
\hline 269. & Erigeron acris L. & Ep & $\mathrm{K}$ & $\mathrm{H}$ & rare \\
\hline 270. & E. annuus (L.) Pers. & Ep & $\mathrm{K}$ & $\mathrm{H}$ & frequent \\
\hline 271. & Conyza canadensis (L.) Cronquist & Ep & $\mathrm{K}$ & $\mathrm{T}$ & common \\
\hline 272. & Filago minima $(\mathrm{Sm}$.$) Pers.$ & Aps & $\mathrm{K}$ & $\mathrm{T}$ & rare \\
\hline 273. & Gnaphalium sylvaticum L. & $\mathrm{Al}$ & $\mathrm{W}$ & $\mathrm{H}$ & rather rare \\
\hline 274. & G. uliginosum L. & Anw & $\mathrm{K}$ & $\mathrm{T}$ & quite common \\
\hline 275. & Helichrysum arenarium /L./ Moench & Aps & W & $\mathrm{H}$ & very rare \\
\hline 276. & Bidens frondosa $\mathrm{L}$. & $\mathrm{Kn}$ & $\mathrm{K}$ & $\mathrm{T}$ & rare \\
\hline 277. & B. tripartita $\mathrm{L}$. & Anw & $\mathrm{K}$ & $\mathrm{T}$ & rather rare \\
\hline 278. & Iva xantifolia Nutt. & Ep & $\mathrm{K}$ & $\mathrm{T}$ & very rare \\
\hline 279. & Galinsoga parviflora Cav. & Ep & $\mathrm{K}$ & $\mathrm{T}$ & common \\
\hline 280. & G. ciliata /Raf./S.F. Blake & Ep & $\mathrm{K}$ & $\mathrm{T}$ & quite common \\
\hline 281. & Anthemis cotula $\mathrm{L}$. & $\mathrm{Ar}$ & $\mathrm{K}$ & $\mathrm{T}$ & very rare \\
\hline 282. & A. arvensis $\mathrm{L}$. & $\mathrm{Ar}$ & $\mathrm{K}$ & $\mathrm{T}$ & quite common \\
\hline 283. & A. nobilis $\mathrm{L}$. & Ef & $\mathrm{K}$ & $\mathrm{T}$ & very rare \\
\hline 284. & Achillea millefolium $\mathrm{L}$. & Ał & $\mathrm{W}$ & $\mathrm{H}$ & common \\
\hline 285. & Matricaria maritima subsp inodora Schulc-Bip. & Ar & $\mathrm{K}$ & $\mathrm{T}$ & very common \\
\hline 286. & Chamomilla recutita (L.) Rauschert & Ar & $\mathrm{K}$ & $\mathrm{T}$ & rare \\
\hline 287. & Ch. suaveolens /Pursh/ Rydb. & Ep & $\mathrm{K}$ & $\mathrm{T}$ & frequent \\
\hline
\end{tabular}




\begin{tabular}{|c|c|c|c|c|c|}
\hline 288. & Tanacetum vulgare $\mathrm{L}$. & $\mathrm{Al}(\mathrm{nw})$ & $\mathrm{W}$ & $\mathrm{H}$ & quite common \\
\hline 289. & Leucanthemum vulgare Lam. S. STR & Ał & W & $\mathrm{H}$ & very rare \\
\hline 290. & Artemisia absinthium L. & Ar & $\mathrm{W}$ & $\mathrm{H}$ & rare \\
\hline 291. & A. vulgaris $\mathrm{L}$. & $\mathrm{Al}(\mathrm{nw})$ & $\mathrm{W}$ & $\mathrm{H}$ & common \\
\hline 292. & A. аппиа $\mathrm{L}$. & Ef & $\mathrm{K}$ & $\mathrm{T}$ & very rare \\
\hline 293. & A. campestris $\mathrm{L}$. & Aps & $\mathrm{W}$ & $\mathrm{H}$ & rare \\
\hline 294. & Tussilago farfara $\mathrm{L}$. & Anw & $\mathrm{W}$ & G & rare \\
\hline 295. & Senecio vulgaris L. & $\mathrm{Ar}$ & $\mathrm{K}$ & $\mathrm{T}(\mathrm{H})$ & quite common \\
\hline 296. & S. vernalis Waldst. et Kit. & $\mathrm{Ar}$ & $\mathrm{K}$ & $\mathrm{T}(\mathrm{H})$ & rare \\
\hline 297. & S. jacobea $\mathrm{L}$ & $\mathrm{Al}$ & $\mathrm{W}$ & $\mathrm{H}$ & very rare \\
\hline 298. & Arctium tomentosum Hill. & $\mathrm{Al}$ & $\mathrm{K}$ & $\mathrm{H}$ & rare \\
\hline 299. & A. lappa $\mathrm{L}$ & $\mathrm{Al}$ & $\mathrm{K}$ & $\mathrm{H}$ & rare \\
\hline 300. & A minus /Hill/ Bernh. & $\mathrm{Ar}$ & $\mathrm{K}$ & $\mathrm{H}$ & very rare \\
\hline 301. & Carduus acanthoides L. & $\mathrm{Ar}$ & $\mathrm{K}$ & $\mathrm{H}$ & very rare \\
\hline 302. & C. crispus $\mathrm{L}$. & Ep & $\mathrm{K}$ & $\mathrm{H}$ & very rare \\
\hline 303. & Cirsium vulgare (Savi) Ten. & Ał & $\mathrm{W}$ & $\mathrm{H}$ & very rare \\
\hline 304. & C. arvense /L./ Scop. & $\mathrm{Al}$ & $\mathrm{W}$ & G & very common \\
\hline 305. & Centaurea cyanus L. & $\mathrm{Ar}$ & $\mathrm{K}$ & $\mathrm{T}$ & very common \\
\hline 306. & C. scabiosa $\mathrm{L}$. & Amk & $\mathrm{W}$ & $\mathrm{H}$ & very rare \\
\hline 307. & C. stoebe $\mathrm{L}$. & Amk & $\mathrm{K}$ & $\mathrm{H}$ & very rare \\
\hline 308. & C. jacea $\mathrm{L}$. & Ał & $\mathrm{W}$ & $\mathrm{H}$ & rare \\
\hline 309. & Cichorium intybus $\mathrm{L}$. & Ar & $\mathrm{W}$ & G & rather rare \\
\hline 310. & Arnoseris minima /L./ Schweigg. \& Körte & Aps & $\mathrm{K}$ & $\mathrm{T}$ & frequent \\
\hline 311. & Hypochoeris radicata $\mathrm{L}$. & Ał & $\mathrm{W}$ & $\mathrm{H}$ & rare \\
\hline 312. & H. glabra L. & Aps & $\mathrm{K}$ & $\mathrm{T}$ & rare \\
\hline 313. & Leontodon autumnalis $\mathrm{L}$. & Ał & $\mathrm{W}$ & $\mathrm{H}$ & rather rare \\
\hline 314. & Tragopogon pratensis L. S. STR & Ał & $\mathrm{W}$ & $\mathrm{H}$ & very rare \\
\hline 315. & Sonchus oleraceus L. & $\mathrm{Ar}$ & $\mathrm{K}$ & $\mathrm{T}(\mathrm{H})$ & quite common \\
\hline 316. & S. asper /L./ Hill. & $\mathrm{Ar}$ & $\mathrm{K}$ & $\mathrm{T}$ & common \\
\hline 317. & S. arvensis $\mathrm{L}$. & Anw & $\mathrm{W}$ & $\mathrm{H}$ & common \\
\hline 318. & Lactuca serriola $\mathrm{L}$ & $\mathrm{Ar}$ & $\mathrm{K}$ & $\mathrm{H}$ & rare \\
\hline 319. & Taraxacum sp. F.H. Wigg. coll. & Ał & $\mathrm{W}$ & $\mathrm{H}$ & quite common \\
\hline 320. & Lapsana communis $\mathrm{L}$. & $\mathrm{Al}$ & $\mathrm{K}$ & $\mathrm{T}(\mathrm{H})$ & quite common \\
\hline 321. & Crepis tectorum $\mathrm{L}$. & Aps & $\mathrm{K}$ & $\mathrm{T}$ & rather rare \\
\hline 322. & C. capillaris (L.) Wallr. & $\mathrm{Al}$ & $\mathrm{K}$ & $\mathrm{T}$ & rare \\
\hline 323. & Hieracium pilosella $\mathrm{L}$. & Aps & $\mathrm{W}$ & $\mathrm{H}$ & rather rare \\
\hline \multirow[t]{2}{*}{324.} & H. umbellatum $\mathrm{L}$. & Ał & $\mathrm{W}$ & $\mathrm{H}$ & very rare \\
\hline & Liliaceae & & & & \\
\hline 325. & Allium vineale $\mathrm{L}$. & Amk & $\mathrm{W}$ & G & rare \\
\hline 326. & Gagea pratensis (Pers.) Dumort & At & $\mathrm{W}$ & $\mathrm{G}$ & rare \\
\hline \multicolumn{6}{|c|}{ Juncaceae } \\
\hline 327. & Juncus capitatus Weigel. & Anw & $\mathrm{K}$ & $\mathrm{T}$ & rare \\
\hline 328. & J. bufonius L. & Anw & $\mathrm{K}$ & $\mathrm{T}$ & frequent \\
\hline 329. & Luzula campestris /L./ DC. & At & $\mathrm{W}$ & $\mathrm{H}$ & very rare \\
\hline \multicolumn{6}{|c|}{$\begin{array}{r}\text { Poaceae } \\
\end{array}$} \\
\hline 330. & Festuca pratensis Huds. & Ał & $\mathrm{W}$ & $\mathrm{H}$ & rare \\
\hline 331. & F. rubra L. s.s & Al & $\mathrm{W}$ & $\mathrm{H}$ & very rare \\
\hline 332. & F. ovina $\mathrm{L}$. & Aps & $\mathrm{W}$ & $\mathrm{H}$ & very rare \\
\hline 333. & Lolium perenne $\mathrm{L}$. & Ał & $\mathrm{W}$ & $\mathrm{H}$ & rather rare \\
\hline 334. & L. multiflorum Lam. & Ep & $\mathrm{K}$ & $\mathrm{H}$ & very rare \\
\hline
\end{tabular}




\begin{tabular}{|c|c|c|c|c|c|}
\hline 335. & Poa annua $\mathrm{L}$. & Ał & $\mathrm{K}$ & $\mathrm{T}$ & quite common \\
\hline 336. & P. pratensis $\mathrm{L}$. & Ał & W & $\mathrm{H}$ & very rare \\
\hline 337. & P. palustris L. & Anw & W & $\mathrm{H}$ & very rare \\
\hline 338. & Dactylis glomerata L. & Ał & W & $\mathrm{H}$ & rare \\
\hline 339. & Apera spica-venti (L.) P. Beauv. & $\mathrm{Ar}$ & $\mathrm{K}$ & $\mathrm{T}$ & common \\
\hline 340. & Briza media $\mathrm{L}$. & Ał & W & $\mathrm{H}$ & very rare \\
\hline 341. & Bromus tectorum $\mathrm{L}$. & Ar & $\mathrm{K}$ & $\mathrm{T}$ & very rare \\
\hline 342. & B. sterilis L. & $\mathrm{Ar}$ & $\mathrm{K}$ & $\mathrm{T}$ & very rare \\
\hline 343. & B. inermis Leyss. & Amk & $\mathrm{W}$ & $\mathrm{H}$ & rare \\
\hline 344. & B. secalinus L. & $\mathrm{Ar}$ & $\mathrm{K}$ & $\mathrm{T}$ & frequent \\
\hline 345. & B. hordeaceus L. & Ał & W & $\mathrm{H}$ & rare \\
\hline 346. & Elymus repens /L./ Gould. & Anw & $\mathrm{W}$ & G & common \\
\hline 347. & E. hispidus (Opiz) Melderis & Ef & $\mathrm{W}$ & G & very rare \\
\hline 348. & Avena fatua $\mathrm{L}$. & $\mathrm{Ar}$ & $\mathrm{K}$ & $\mathrm{T}$ & quite common \\
\hline 349. & A. strigosa $\mathrm{L}$. & Ar & $\mathrm{K}$ & $\mathrm{T}$ & rather rare \\
\hline 350. & $\begin{array}{l}\text { Arrhenantherum alatius (L.) P. Baeur. ex J. Presl } \\
\& \text { C. Presl }\end{array}$ & Ał & $\mathrm{W}$ & $\mathrm{H}$ & very rare \\
\hline 351. & Anthoxanthum aristatum Boiss. & Ep & $\mathrm{K}$ & $\mathrm{T}$ & frequent \\
\hline 352. & Holcus lanatus L. & Ał & W & $\mathrm{H}$ & rare \\
\hline 353. & H. mollis L. & $\mathrm{Al}$ & W & $\mathrm{H}$ & very rare \\
\hline 354. & Corynephorus canescens (L.) P. Beauv. & Aps & $\mathrm{W}$ & $\mathrm{H}$ & very rare \\
\hline 355. & Agrostis capillaris $\mathrm{L}$. & Ał & $\mathrm{W}$ & $\mathrm{H}$ & rare \\
\hline 356. & A. stolonifera $\mathrm{L}$. & Anw & $\mathrm{W}$ & $\mathrm{H}$ & rather rare \\
\hline 357. & Phleum pratense $\mathrm{L}$. & Ał & $\mathrm{W}$ & $\mathrm{H}$ & rather rare \\
\hline 358. & Alopecurus geniculatus L. & Anw & $\mathrm{W}$ & $\mathrm{H}$ & rare \\
\hline 359. & A. pratensis $\mathrm{L}$. & Ał & $\mathrm{W}$ & G & very rare \\
\hline 360. & Phragmites australis /Cav./ Trin. ex Steud. & Anw & W & G & frequent \\
\hline 361. & Nardus stricta L. & Aps & W & $\mathrm{H}$ & very rare \\
\hline 362. & Echinochloa crus-galli (L.) P. Beauv. & Ar & $\mathrm{K}$ & $\mathrm{T}$ & common \\
\hline 363. & Digitaria ischaemum (Scheb.) H. L. Mühl. & $\mathrm{Ar}$ & $\mathrm{K}$ & $\mathrm{T}$ & rather rare \\
\hline 364. & D. sanguinalis $(\mathrm{L}$.$) Scop.$ & $\mathrm{Ar}$ & $\mathrm{K}$ & $\mathrm{T}$ & rare \\
\hline 365. & Setaria pumila (Poir) Roem.Schult. & Ar & $\mathrm{K}$ & $\mathrm{T}$ & common \\
\hline 366. & S. viridis (L.) P. Beauv. & $\mathrm{Ar}$ & $\mathrm{K}$ & $\mathrm{T}$ & frequent \\
\hline
\end{tabular}

366. S. viridis (L.) P. Beauv.

367. Carex hirta L.
Ał
W
rare

Explanations: Ar-archeophytes, Ep-epecophytes, Er-ergasiophytes, A-apophytes: 1-meadows, nw-waterside, ps-psammophilous grassland, mk - xerothermic grassland, 1 - forest, $\mathrm{z}$ - shrub; K - short-lived, W - perennial, $\mathrm{T}$ - therophytes, $\mathrm{H}$-hemicryptophytes, G - geophytes, Ch - chamaephytes,

Common species are the least numerous group. Only 13 species were found in cereals, tuber crops, and stubble fields of the Middle Vistula River Valley, that is, $3.5 \%$ of the segetal flora. These species are as follows: Equisetum arvense, Fallopia convolvulus, Polygonum aviculare, Chenopodium album, Stellaria media, Viola arvensis, Convolvulus arvensis, Myosotis arvensis, Matricaria maritima subsp. inodora, and Centaurea cyanus. It is worth noticing that the following taxons: Bromus secalinus, Melandrium noctiflorum, Consolida regalis, Aethusa cynapium, Chaenorhinum minus, Veronica agrestis, Veronica polita and Agrostemma githago, are sporadic species in many regions of Poland, whereas in the study area they are frequently found. The group of quite common species was made up of 6 taxons established on soils which are wet and excessively wet due to a high level of groundwater. They include the following: Stachys palustris, Mentha arvensis, Rorippa sylvestris, Plantago intermedia, Gnaphalium uliginosum, and Polygonum amphibium.

The analysis of taxons in terms of their geographical and historical classification revealed 227 $(62 \%)$ of native species and $140(38 \%)$ species which were newcomers during different historical periods (Fig. 2). 


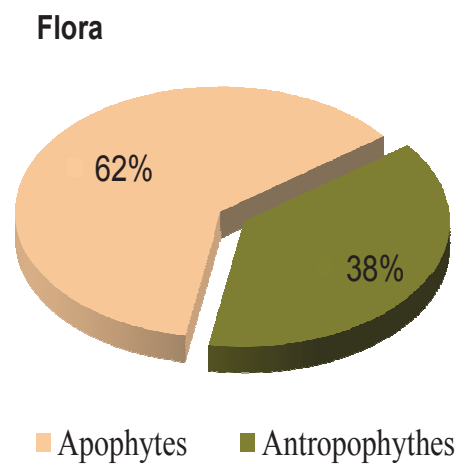

Fig. 2. Percentage proportion of geographical and historical groups in the segetal flora of the mesoregion of the Middle Vistula River Valley.

The apophytes were dominated by meadow apophytes -78 species (35\%), followed by waterside apophytes and apophytes established in excessively wet habitats -48 species (21\%) (Fig. 3). These groups were present in more than half of all the native species, as they became established close to meadow communities and in close proximity to the river contributing to the growth and development of moisture-loving species, deep-rooting plants in particular. The following taxons were less abundant: forest and bush taxons (39 species $-17 \%$ ), sandy, sandy grassland and dune taxons (32 species - 14\%), and xerothermic grassland taxons (30 species $-13 \%)$.

\section{Apophytes}

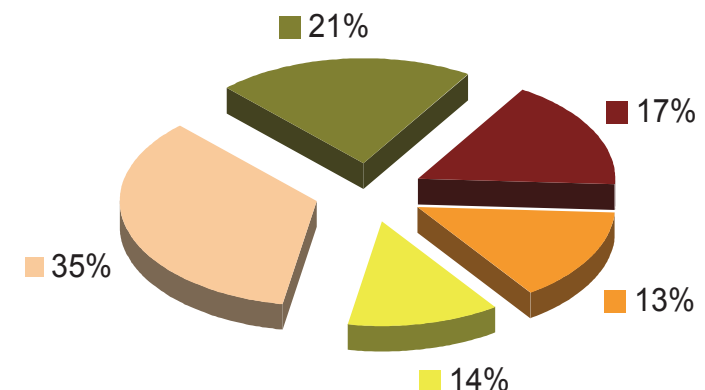

meadows habitats

forest and bushes

xerothermic grassland

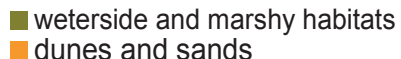

Fig. 3. Percentage proportion of apophytes in the segetal flora of the mesoregion of the Middle Vistula River Valley

Common apophytes in the study area were as follows, e.g.: Sonchus arvensis, Plantago intermedia, Rorippa sylvestris, Stachys palustris, Mentha arvensis, Equisetum arvense, and Elymus repens. Rare apophytes included the following: Gagea pratensis,
Juncus capitatus, Radiola linoides, Peplis portula, Sherardia arvensis, Galeopsis speciosa, Hypericum humifusum, and Ranunculus sardous. The above-mentioned species are rare in Poland and are classified as threatened species.

The anthropophytes were dominated by archeophytes - 97 species (69\%) (Fig. 4). Epecophytes and ergasiophygophytes were much less frequent 23 and 15 species, respectively (16 and $11 \%$ ). Moreover, 5 ephemerophytes were found (4\%). The following archeophytes were the most common: Scleranthus annuus, Papaver rhoeas, Lathyrus tuberosus, Euphorbia helioscopia, Anagallis arvensis, Myosotis arvensis, Lamium amplexicaule, Lamium purpureum, Sonchus arvensis, Sonchus oleraceus, and Sonchus asper.

\section{Antropophythes}

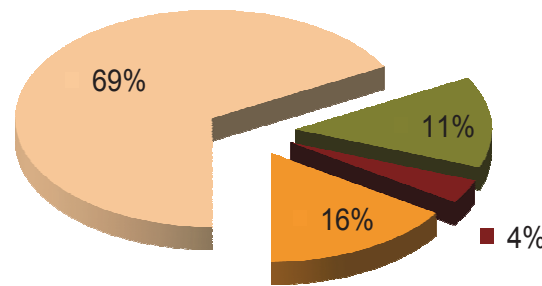

Archeophytes $\square$ Ergasiophytes

- Efemerophytes Epecophytes

Fig. 4. Percentage proportion of individual groups of anthropophytes in the segetal flora of the Middle Vistula River Valley.

Rare and very rare archeophytes included: Centunculus minimus, Fumaria vaillantii, Aphanes arvensis, Geranium dissectum, Valerianella dentata, Valerianella rimosa, Neslia paniculata, and Camelina sativa. The aforementioned species are also rarely encountered in other regions in Poland. The following epecophytes were found: Amaranthus retroflexus, Oxalis fontana, Rorippa austriaca, Cuscuta campestris, Iva xantifolia, Bidens frondosa, Elsholtzia ciliata, Anthoxanthum aristatum, and others.

The analysis of the biological spectrum of the segetal flora showed that therophytes were the dominant life forms. The segetal flora included 185 therophytes (50\%), 143 hemicryptophytes (39\%), 37 geophytes $(10 \%), 1$ hydrophyte $(0.5 \%)$, and 1 chamaephyte $(0.5 \%)$ (Fig. 5). When the biological types of native species were analysed, it was found that hemicryptophytes were the dominant life form (54\%). Therophytes were dominant anthropophytes (83\%). 


\section{Flora}

$\square$ Terophytes $\backsim$ Hemicryptophytes $\backsim$ Chameophytes $\|$ Geophytes $\backsim$ Hydrophytes

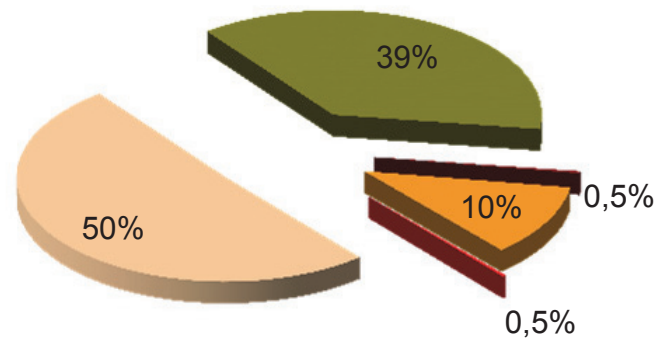

\section{Apophytes}

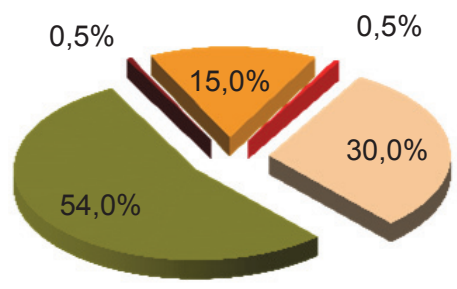

\section{Antropophytes}

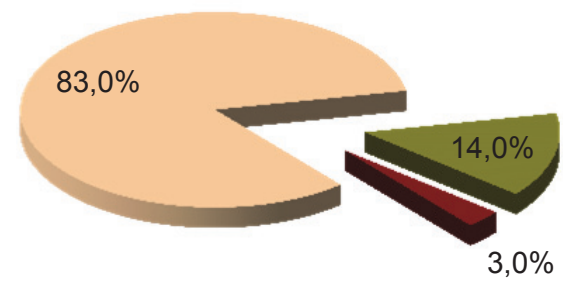

Fig. 5. Percentage proportion of forms in the segetal flora of the Middle Vistula River Valley.

Non-perennial (short-lived) species were more abundant than perennials. Of the 370 flora species in the study area, 206 taxons were non-perennials (56\%) and 161 taxons were perennials (44\%) (Fig. 6). Moreover, perennial apophytes and non-perennial anthropophytes were more abundant ( 65 and $90 \%$, respectively).

\section{Flora}

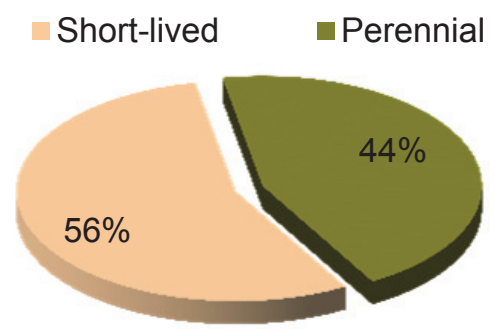

\section{Apophytes}

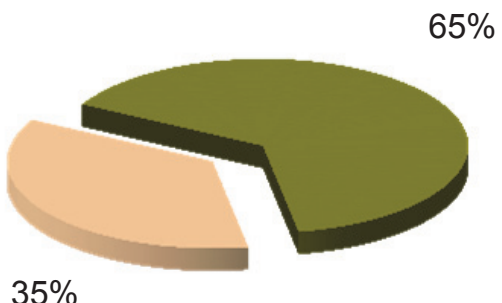

Antropophythes

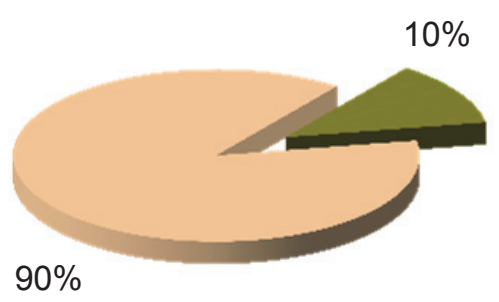

Fig. 6. Percentage proportion life of species in the segetal flora of the Middle Vistula River Valley.

\section{DISCUSSION}

Segetal flora is becoming more and more impoverished mainly due to increasing intensification of agricultural production, particularly in western and central Europe [2,3,16-21].
The segetal flora in the Middle Vistula River Valley is very abundant. A total of 367 vascular species were found in cereals, tuber crops, and stubble fields. The flora in the study area seems to be much more abundant than in Mazovia [6], the Mazovian Landscape Park [22] or the Kampinos National Park 
[23]. When compared with the rest of Poland, the study area has fewer species than the area of the PrzeborskoMałopolskie Range - 393 species [24] and the Piotrków Plain - 374 species [25].

Such floristic abundance was due to the fact that the study area is located along the banks of the Vistula River. A wide variety of habitats are found there, the dominant ones being fertile alluvial soils as well as acid and dry habitats. Also, the fields are fragmented and cultivated in quite a traditional way.

The analysis of the segetal flora demonstrated that apophytes were more numerous that anthropophytes. Such findings have been reported for other regions in Poland [6,13,22,25-27]. The opposite has been reported only for the Vistula delta area (Źuławy Wiślane) has [28]. According to D ą b k ow sk a and Sygulska [29], the fact that native species dominate over newcomers from different historical periods is indicative of a small effect of human pressure and slight transformation of segetal flora. Over 58\% archeophytes of 165 taxons occurring in Poland [30] were found in the study area. Such a high share of this group of species in the flora of the Middle Vistula River Valley may be indicative of the dominance of traditional farming in the area. It has been reported in European literature that this group of species is receding due to the application of herbicides, the use of certified seeds for sowing, the abandonment of certain cultivation practices, the exclusion from cultivation of poor quality fields with infertile acid soils and oligotrophic carbonate-rich habitats, and changing the ways in which fields are used [2,17,31]. Under Polish conditions, the fragmentation of fields may be an additional factor as it contributes to less competition with native species. In recent years, agrocenoses have also been effected by the increasing proportion of foreign invasive species which may threaten the native flora [32-35]. The epecophytes that had a special influence on the composition and structure of communities in the study area included the following: Oxalis fontana, Veronica persica, Conyza canadensis, Galinsoga parviflora, and Anthoxanthum aristatum.

\section{Acknowledgements}

Research supported by the Ministry of Science and Higher Education of Poland as part of the statutory activities of the Department of Agricultural Ecology, Siedlce University of Natural Sciences and Humanities.

\section{REFERENCES}

1. Kornaś J. Chwasty polne rozprzestrzeniane z materiałem siewnym. Specjalizacja ekologiczna i procesy wymierania. Zesz Nauk AR Krakow. 1987; 19: 23-36.
2. Stehlik I, Caspersen JP, Wirth L, Holderegger R. Floral free fall in the Swiss lowlands: environmental determinants of local plant extinction in a periurban landscape. J Ecol. 2007; 95(4): 734-744. http://dx. doi.org/10.1111/j.1365-2745.2007.01246.x

3. Skrajna T, Gozdowski B, Ługowska M. The transformations of field communities with Illecebrum verticillatum L. (Caryophyllaceae) on the borderlands of its European range (central-eastern Poland). Pol J Ecol. 2014; 62(1): 3-15.

4. Warcholińska AU, Siciński J T. Ekspansja Anthoxanthum aristatum Boiss. w środkowej Polsce. Zesz Nauk Akad Tech Roln Bydg. 1996; 196(38): 183-192.

5. Tokarska-Guzik B. The establishment and spread of alien plant species (Kenophytes) in the flora of Poland. Pr Nauk Uniw Śląskiego. 2005; 2372: 1-192.

6. Wójcik Z. Udział apofitów i antropofitów w zbiorowiskach segetalnych Mazowsza. Mat Zakł Fitosoc Stos UW. 1968; 25: 109-122.

7. Kondracki J. Geografia regionalna Polski. PWN. Warszawa: 2002; 3: 11-194

8. Woś A. Klimat Polski. Warsaw: Polish Scientific Publishers PWN; 1999.

9. Rutkowski L. Klucz do oznaczania roślin naczyniowych Polski niżowej. Warsaw: Polish Scientific Publishers PWN; 1998.

10. Mirek Z, Piękoś-Mirkowa H, Zając A, $\mathrm{Z}$ ają c $\mathrm{M}$, editors. Flowering plants and pteridophytes of Poland - a checklist. Cracow: W. Szafer Institute of Botany, Polish Academy of Sciences; 2002. (Biodiversity of Poland; vol 1).

11. Zają c A. Pochodzenie archeofitów występujących w Polsce. Rozpr Hab UJ Kraków. 1979; 29: 5-213.

12. Jackowiak B. Antropogeniczne przemiany flory roślin naczyniowych Poznania. Wyd Nauk UAM Pozn. Biol. 1990; 42: 1-232.

13. Korniak T. Flora segetalna północno-wschodniej Polski, i jej przestrzenne zróżnicowanie i współczesne przemiany. Acta Acad Agric Tech Olst Agric. 1992; 53(A suppl): 5-76.

14. Rothmaler W. Exkursionsflora von Deutschland. G. Fischer Verlag; Jena. 2000; 3: 1-734.

15. Jas iewicz A. Rośliny naczyniowe Bieszczadów Zachodnich. Mongr Bot. 1965; 20: 1-340

16. Pyšek P, Jarošík V, K ropáč Z, Chytrý M, Wild J, Tichý L. Effects of abiotic factors on species richness and cover in Central European weed communities. Agric Ecosyst Env. 2005; 109(1-2): 1-8. http://dx.doi. org/10.1016/j.agee.2005.02.018

17. Meyer S, Wesche K, Krause B, Leuschner C. Dramatic losses of specialist arable plants in Central Germany since the 1950s/60s - a cross-regional analysis. Divers Distrib. 2013; 19(9): 1175-1187. http://dx.doi.org/10.1111/ ddi. 12102

18. Storkey J, Meyer S, Still KS, Leuschner C. The impact of agricultural intensification and land-use change on the European arable flora. Proc R Soc B. 
2012; 279(1732): 1421-1429. http://dx.doi.org/10.1098/ rspb.2011.1686

19. Májeková J, Zaliberová M, Šibík J, Klimová K. Changes in segetal vegetation in the Borská nížina Lowland (Slovakia) over 50 years. Biologia (Bratisl). 2010; 65(3): 465-478. http://dx.doi.org/10.2478/s11756-0 $10-0035-5$

20. Skrzyczyńska J, Ługowska M. Rzadkie gatunki segetalne wschodniej części Doliny Środkowej Wisły. Fragm Flor Geobot Pol. 2006; 13(1): 11-15.

21. Szczęśniak E, Dajdok Z, Kącki Z. Metodyka oceny zagrożenia i kategoryzacja zagrożonych archeofitów na przykładzie Dolnego Śląska. Acta Bot Siles. 2011; 1: 9-28.

22. Skrajna T, Skrzyczyńska J, Ługowska M. The segetal flora of the Mazowiecki Landscape Park. Plant Breed Seed Sci. 2010; 61: 93-104. http://dx.doi.org/ 10.2478/v10129-010-0016-4

23. Bomanowska A. Wybrane cechy flory segetalnej Kampinowskiego Parku Narodowego. Pam Puł. 2006; 143: 27-35.

24. Wnuk Z. Flora segetalna Pasma PrzedborskoMałogoskiego i przyległych terenów. Acta Univ Lodz. 1978; 11(20): 183-255.

25. Warcholińska AU. Flora segetalna Równiny Piotrkowskiej (Mezoregion Nizin Środkowopolskich). Zesz Nauk UŁ Ser II. 1976; 8: 63-95.

26. Skrzyczyńska J. Studia nad florą i zbiorowiskami Wysoczyzny Siedleckiej Rozpr. hab. 1994; 39: 1-145

27. Skrzyczyńska J, Rzymowska Z. The segetal flora of the Bug river geomorphological territories in Podlasie region. Acta Agrobot. 2001; 54(1): 115-135. http://dx.doi. org/10.5586/aa.2001.011

28. Hołdyński C. Flora segetalna, zróżnicowanie florystyczno-ekologiczne i przemiany szaty roślinnej pól uprawnych w aktualnych warunkach agroekologicznych Żuław Wiślanych. Acta Acad Agric Tech Olst Agric. 1991; 51(403): 1-50.

29. Dąbkowska T, Sygulska P. Variations in weed flora and the degree of its transformation in ecological and extensive conventional cereal crops in selected habitats of the Beskid Wyspowy Mountains. Acta Agrobot. 2013; 66(2): 123-136. http://dx.doi.org/10.5586/aa.2013.029

30. Zając M, Zając A, Tokarska-Guzik B. Extinct and endangered archaeophytes and the dynamics of their diversity in Poland. Biodiv Res Conserv. 2010; 13: 17-24. http://dx.doi.org/10.2478/v10119-009-0004-4
31. Preston CD, Pearman DA, Hall AR. Archaeophytes in Britain. Bot J Linn Soc. 2004; 145(3): 257-294. http://dx.doi.org/10.1111/j.1095-8339.2004.00284.x

32. L o s o s ova Z, Si mon ova D. Changes during the 20 th century in species composition of synanthropic vegetation in Moravia (Czech Republic). Preslia. 2008; 80: 291-305.

33. Pál R. Invasive plants threaten segetal weed vegetation of south Hungary. Weed Technol. 2004; 18: 1314-1318. http://dx.doi.org/10.1614/0890-037X(2004)018[1314:IPTS WV]2.0.CO;2

34. Pinke G, Pal R, Király G, Szendrôdi V, Esterhaázy A. The occurrence and habitat conditions of Anthoxanthum puelii Lecoq; Lamotte and other AtlanticMediterranean weed species in Hungary. J Plant Prot. 2006; 22: 587-596.

35. Tokarska-Guzik B, Węgrzynek B, Urbisz A, Urbisz A, Nowak T, Bzdęga K. Alien vascular plants in the Silesian Upland of Poland: distribution, patterns, impacts and threats. Biodiv Res Conserv. 2011; 19: 33-54. http://dx.doi.org/10.2478/v10119-010-0019-x

\section{Flora segetalna Doliny Środkowej Wisły}

\section{Streszczenie}

Celem pracy była charakterystyka flory segetalnej Doliny Środkowej Wisły. W uprawach zbóż jarych i ozimych, w uprawach okopowych oraz na ścierniskach zbożowych zarejestrowano 367 gatunków. Na tak duże bogactwo florystyczne miało wpływ usytuowanie terenu badań w bliskim sąsiedztwie z rzeką, bezpośredni kontakt półnaturalnych zbiorowisk z polami uprawnymi, a także drobnopowierzchniowy rozkład pól z dużym zróżnicowaniem siedlisk. W analizowanej florze odnotowano przewagę apofitów (62\%) nad antropofitami (38\%). Wśród gatunków rodzimych dominowały apofity łąkowe (35\%), natomiast w grupie antropofitów archeofity (69\%). Analiza spektrum życiowego wykazała przewagę terofitów (50\%) nad hemikryptofitami (39\%). We florze segetalnej Doliny Środkowej Wisły gatunki krótkotrwałe stanowiły $56 \%$, a wieloletnie $44 \%$. Duży udział archeofitów (26\%) wśród wszystkich zarejestrowanych gatunków może świadczyć o zachowaniu tradycyjnych form gospodarowania na tym terenie.

Handling Editor: Elżbieta Weryszko-Chmielewska

This is an Open Access digital version of the article distributed under the terms of the Creative Commons Attribution 3.0 License (creativecommons.org/licenses/by/3.0/), which permits redistribution, commercial and non-commercial, provided that the article is properly cited.

CThe Author(s) 2014 Published by Polish Botanical Society 
